\title{
AeLF: Mixing Adaptive Learning System with Learning Management System
}

\author{
Aimad Qazdar \\ GMES Laboratory,ENSA \\ Ibn Zohr University \\ Agadir, Morocco
}

\author{
Chihab Cherkaoui \\ IRF-SIC Laboratory, ENCG \\ Ibn Zohr University \\ Agadir, Morocco \\ Driss Mammass \\ IRF-SIC Laboratory, Faculty of science \\ Ibn Zohr University \\ Agadir, Morocco
}

\author{
Brahim Er-Raha \\ GMES Laboratory,ENSA \\ Ibn Zohr University \\ Agadir, Morocco
}

\begin{abstract}
Several educational institutes use e-Learning platforms especially Learning Management System (LMSs) to manage the learning process. Adaptive Learning System (ALSs) supports the learning process using adaptive guidance and possibly personnalized content. The AeLF offers a new framework that brings the two systems together. This is done by a single sign-on, a User Model, an Experience Learner Tracker, a Domain Model and an Adaptive Engine. Authors can create structured course material and define the pedagogical rules through a graphical interface. The experiences learner tracker can detect all the interaction learner/system and a supple adaptation engine offers almost any type of adaptation a learner might want. This article presents the architecture of the AeLF environment, and describes the all its components.
\end{abstract}

\section{Keywords:}

Adaptation, adaptive learning systems, adaptive learning management system, learning content, adaptation engine

\section{INTRODUCTION}

Toward the last twenty years, two complementary technologies have been introduced in the world of the modern learning: e-Learning platforms (LMS ${ }^{1}$ and MOOC $^{2}$ ) and Adaptive Learning Systems (ALS).

LMS (such as Moodle ${ }^{3}$ Blackboard ${ }^{4}$ Saka ${ }^{5}$, etc.) is an integrated system which supports the learning process and administration. It presents an important package of services and features enhancing e-Learning. An LMS supports teachers in developing e-learning courses that students can enroll in. In addition, it allows the use

\footnotetext{
${ }^{1}$ Learning Management System

${ }^{2}$ Massive Open Online Course

${ }^{3}$ http://www.moodle.org/

${ }^{4}$ http://www.blackboard.com/

${ }^{5}$ https://www.sakaiproject.org/
}

of external learning materials (resources/activities) and the reuse of already developed learning materials. An LMS can support creating and serving tests, grading assignments, publishing course material.It can also allow a synchronous and /or an asynchronous communication between users (teachers, students or administrators) through chat rooms, discussion forums, etc.

Nevertheless this category of systems doesnt support the adaptation and personalization of courses; typically they present exactly the same course for every learner without consideration of the learners individual characteristics, situation, and needs[16]. Such a one-size-fits-all approach often leads to frustration, difficulties in learning, and a high dropout rate [23]|34]

ALS supports the learning itself, by means of personalized access to course material. It allows selecting dynamically the most relevant learning object in order to present them at the right time and in the right direction for every learner. By taking individual learning differences and contexts into account, the ALS can improve learning outcomes, require less effort, reduce time required, and result in higher learner satisfaction. An ALS can, for example, adapt learning material/activities to a learners prior knowledge [7] [36], preferred learning style [33] [28] [17], affective states [35][13], and so on. Furthermore, this system can take advantage of nearby objects or people who might be able to help in the learning process [14][25], consider the characteristics of the learners environment, and take into account the features of the device a learner is using [21].

LMS is most advantageous for the institute (university, company), whereas ALS is most advantageous for the learner.But the AeLF (Adaptive e-Learning Framework) brings the world of LMSs and ALSs together in order to offer a powerful life-long e-learning solution. The AeLF is based on our experience with ALS-CP [1] developed in IRF-SIC Laboratory at the Faculty of science in Ibn Zohr University Agadir (Morocco). This new system allows integrating into LMS the main features of adaptation and personalization used in the ALS. In this paper, the all architecture of AeLF will be presented, including a description of each components of 
its system and a full explanation of how adaptation can be brought about in the LMS using the AeLF.

This paper is outlined as follow: In the beginning, the background of Adaptive systems and the difference between AeLF and other previous systems will be presented. Afterwards, we explore the architecture of AeLF and we describe all its components and the capability of this work to achieve the adaptativity required in LMS. Finally the conclusion and the planned future work are presented in the last section.

\section{OVERVIEW OF ADAPTIVITY IN LEARNINIG ENVIROMMENTS}

This part will be devoted to the presentation of a general overview of adaptation in online learning environments. Then we will list the different approaches explored in the literature followed to make the LMS an adaptive systems.

\subsection{Adaptivity in learning enviromments}

The crucial criterion to providing a responsive learning environment that engages motivates and inspires learners, and through this leads to higher learner satisfaction, is Adaptation.

Adaptation and personnalization, allows the system to present the most relevant and interesting learning objet, while it avoids learning materials that only deal with knowledge the learner already acquired, or that is outside the learner's reach at the current time (but it may display that material later, after the learner has learned the necessary prerequisite knowledge). Educational institutes that use learning environments realize more and more that personalization is a key to creating acceptance of the technology for Technology Enhanced Learning (TEL) by the users.

Personnalization in courses is attained through adaptive learning technologies. These latter provide an environment that intelligently adjusts and adapts to a learners needs by offering pertinent information, instructional materials, feedback and recommendations founded on ones unique individual characteristics and situation [15]. Research has brought forward several adaptive learning solutions.

If we look at the wide field of adaptive learning solution, which are not only designed for adaptive lessons but for other adaptive applications as well. The most famous systems include ELM-ART[5], KBS-Hyperbook [20],AHA![4][10], Interbook[7], GRAPPLE[9][11] and APeLS[8].

The outstanding ELM-ART (Episodic Learner Model, the Adaptive Remote Tutor) adaptive Lisp course for Brusilovsky has not only traced the way for further developments but also became a standard for adaptive link annotations through a traffic light metaphor. By using green, red, grey and yellow colors the ELMART can indicate the recommendation of links to content pages. Many later systems, including AeLF have inherited this metaphor.

Instead of a single Lisp course offered by ELM-ART Brusilovsky has created Interbook for providing a platform that could be used for various courses. An author would essentially redact text content in Microsoft Word which is used as the authoring tools for Interbook. Each outcome concept is associated with a fragment of text (paragraphs or sections) which would be marked as being a prerequisite for other concepts. The content writing in Word will be converted into an adaptive e-learning course just in a press of a button.

The system adaptivity is always based on information about all its users. For this reason the user modeling is a key to success of any adaptive system. Practically user modeling is founded on rules that translate user actions in to user information which can be exploited later. Visiting a course page, to read a text or watch a video, means that you study a concept. That information can be confirmed through a test. Its also used to confirm that you fill prerequisites for learning other concepts. We will see this in AeLF as well.

Knowledge levels of short concepts also totalize to knowledge about chapters and whole courses. Its as if the knowledge of a chapter is being built from the knowledge of underlying sections, and knowledge of the sections being built from the knowledge of the paragraphs or the underlying pages. This approach may provide the false impression that an adaptive system certainly recognizes what goes on in the users mind, with absolute certitude.

However another approach to user modeling, via Bayesian Networks is taken by KBS-Hyperbook for example. The interactions user/system amend the confession of the system that the user acquired certain knowledge. To deal with positive / negative signs of the users knowledge, preferences, interests or any other type of information in Bayesian Networks is easy even in systems that simply employ event-condition-action rules to update knowledge progress.

In the latest versions of AHA! (Adaptive Hypermedia Architecture) an author can define arbitrary adaptation rules and arbitrary presentation. Arbitrary adaptation rules in which the user model can update the rules allows using any model or interpretation of the information about the users. Besides, the content in AHA! is authored in HTML, and also delivered as HTML page contrary to the use of Microsoft Word for authoring in Interbooks. So that authors friendly with HTML can provide an AHA! outcome with any look and feel they desire. So as to manage the complexity of supporting arbitrary presentation and arbitrary adaptation rules AHA! had to distinguish the authoring of the content and presentation from the authoring of the adaptation.

ELM-ART, Interbook and AHA! are examples of systems that support learning process. The learner receives guidance through link annotation and through the conditional fragments. Other support for the process of navigation and the use of services can be found for instance in GRAPPLE and APeLS.

GRAPPLE (Generic Responsive Adaptive Personalized Learning Environment) is a life-long learning solution based on the adaptive hypermedia system AHA!. The GRAPPLE is being developed and integrated with the major LMSs, using a service-oriented architecture approach. The main components of GRAPPLE are GUMF (GRAPPLE User Modeling Framework) which represents the learner in the system, GAT (GRAPPLE Authoring Tool)which is used by teachers to create the conceptual and pedagogical structures of a course- and to associate content with concepts, and the adaptive engine GALE (GRAPPLE Adaptive Learning Environment). The role of the GALE is to provide adaptive content to each learner based on the GUMF and pedagogical rules generated by GAT. 
In the same spirit APeLS (Adaptive Personalized eLearning Service) ,created by Conlan and Wide, is a multi-model, metadata driven adaptive e-learning system that can integrate adaptive fonctionnality in LMS. APeLS puts the content, narrative, and learner into distinct models. The adaptive engine which is used in APeLS is a rule-based engine that provides a model for personalized courses based on a narrative and the learner model.

So far, we havent given a full overview of the introduction of adaptive functionality in online-learning. We have only mentioned a few influential developments. In the next part we will introduce different approches explored in the literature used to integrate ALS in LMS.

\subsection{Adaptivity from ALS to LMS}

Adaptation in online learning environments is often considered as a problem of providing adaptive course sequencing, see e.g. [18] also of supporting by e-learning standards such as SCOORM ${ }^{6}$ and IMS $\mathrm{LD}^{7}$ In the AeLF we approach the question of adaptation from the viewpoint of ALS. Brusilovsky [5][6] defined a taxonomy of adaptive techniques and methodes used in ALS and Paterno 27] updated it, (see [3]). The figure 1 below shows these methods and techniques. Others methods and techniques can be identified from the literature (see c.g [24]), but they are merely variants of the elements presented in this taxonomy. View the lack of space we will

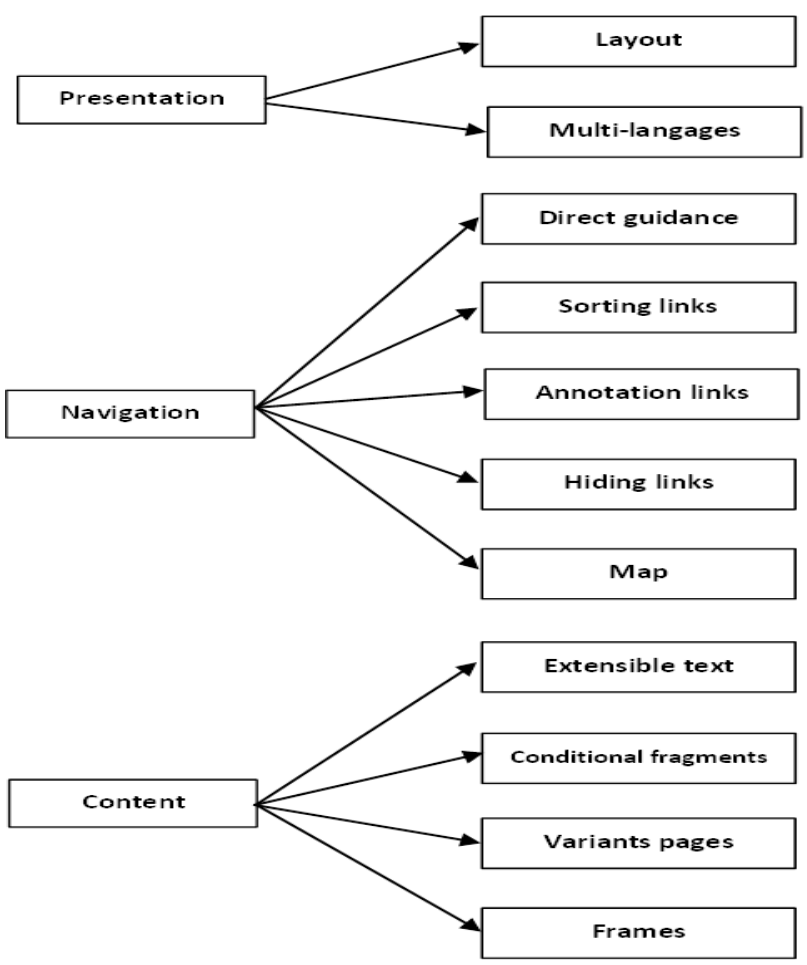

Fig. 1. Methods and techniques of Adaptation in ALS

not explain its all. By cons, we will set out the different approaches

\footnotetext{
$\overline{{ }^{6} \mathrm{http}: / / \text { scorm.com/ }}$

${ }^{7}$ http://www.imsglobal.org/learningdesign/
}

explored in the literatures allowing to integrate these methods and techniques in LMS. These are the following approches :

(1) LMS extensibility,

(2) Modifying LMS source code,

(3) e-Learning Standards,

(4) A framework communicating with LMS (5) LMS customization.

2.2.1 LMS extensibility. The idea of this approche is to incorporate an extension module (plug-in) that can provide features of adaptivity based on the profile of each learner. Several studies have been based on this approach, including the work of the author Sabine Graf [29], Gert Sauerstein [31] and Andre Scherl [2]. Based on this approach, the author Graf [29] integrated sorting adaptive link in the LMS Moodle. The author Sauerstein [31] incorporated annotation links through a traffic light metaphor in Moodle. Finally, Scherl [2] implemented other features namely adaptive navigation map, annotation links and direct guidance also in Moodle.

2.2.2 Modifying LMS source code. This approche is mainly concerns the Open Source LMS. This important feature of this category allows developers to access the source code, read it, understand it and modify it according to the objectives. In this vision, the author Jantke [22] integrated a PHP script to the source code Moodle in order to add some adaptive features to this platform. Among these, we cite the display of adequate resources to the learner or group of learners and the hide of the non adequate ones based on their interactions. Thus, the automatically reassign of learners to groups in response to their individual behaviors and success in the context of learning.

2.2.3 e-Learning Standards. The approach described in this section relates to the use of e-Learning standards and norms, such as: IMS-LD ${ }^{8}$ IMS QTI ${ }^{9}$ SCORM ${ }^{10}$ xAP ${ }^{11}$..etc, as a way to individualize courses for each learner enrolled in LMS. These standards can be operated in two different views. The first point of view is to use the standards as a means for creating adaptive learning scenarios. These can be imported and run in the LMS to deliver learners with individualized learning experiences. This vision is supported by Santos[30]. While the second view affirms that standards are means of information on the experiences and state of learning of each learner. This vision is supported by the author Moisa [26]. Moisa's work consiste to use SCORM and XAPI as a provider of information on the learning progress of each learner. These informations will be processed via adaptive learning algorithms in order to individualize content according to the characteristics of each learner.

2.2.4 A framework communicating with LMS. This approach, which we have adopted in this work, concerne the use of a framework as an adaptivity services producer communicating with the LMS. Adaptation via this approach is not delivered directly from the framework, but it's delivered via the interface of LMS. In literature, the frameworks of adaptation are generally composed of different models communicating each other. Among them, we identify the model of the learner, the domain model and an adaptation engine that reconciles these different models to present an individualized learning path for each student at the time of execution.

\footnotetext{
${ }^{8}$ http://www.imsglobal.org/learningdesign/

${ }^{9}$ http://www.imsglobal.org/question/

${ }^{10}$ http://scorm.com/

${ }^{11} \mathrm{http} / /$ www.adlnet.gov/capabilities/tla/experience-api.html
} 
Communication between the framework and the LMS is provided either via a communication API, web services or http requests. The approach presented in this part was supported by several authors namely Conlan with the framework APeLS [8] and Paul De Bra with the framework GRAPPLE [11].

2.2.5 LMS customization. The main idea of the LMS customization approach is the adaptation of courses in the LMS without the need to develop new modules, or design entirely new solutions. This approach essentially consists of two steps: (i) categorization of learners according to homogeneous groups with respect to different criteria such as: learning style, cognitive level ... etc; (ii) the development of educational resources tailored to each group. Among the authors who have adopted this approach, we quote the author Despotovic-Zrakic[12] and the author Surjono[19].

In the next section we are interested to present the architecture of the proposed system and its capacity to achieve adaptativity in LMS.

\section{AELF:A SOLUTION FOR ADAPTIVE LMS}

In this section, we present the main contributions of the paper. First we clearly demonstrate the infrastructure of the AeLF and then we describe each components of its system. The infrastructure design of the proposed system consists of by five central components Figure 2

This figure show the main components of and the subcomponents of the AeLF. Below, we give detailed descriptions of each components and a clear explanation of its features and interactions between them.

\subsection{The AeLF User Model}

One of the most important questions in adaptive learning is how the system can provide a rich representation of the learner. AeLF uses a common distributed learner model: AUM (AeLF User Model). In order to make learners an actor in the construction of their model, the AUM is open for editing and viewing by both the system and the learners themselves. Therefore it holds information and makes it more reliable and more representative of the learner. Three kind of information are considered in the AUM: personal (name, email address, phone, picture, etc.), preferences (language preferences, favorite colors, the preferred type of educational content, etc.) and Knowledge of learner that is described in relation to each domain model. This component grows dynamically as learners progress in their course. The AUM consists of two main parts:

- Static model: which contains the data indicated by the learner in their first connection to the system. This part can be updated by the learner at any time of their learning throughout a questionnaire delivered by the system.

-Dynamic model: the information stored in is updated only by the AeLF and very dependent on the results and interactions of the learner with the content displayed (by the LMS interface).

As outflow, of the form (Figure 3), an XML file is generated. We propose later a part of an AUM file.

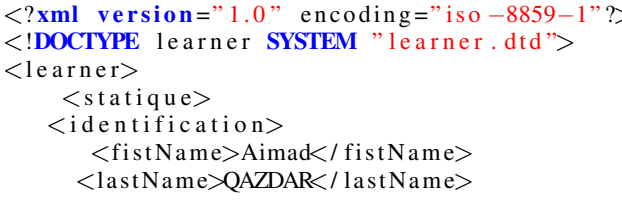

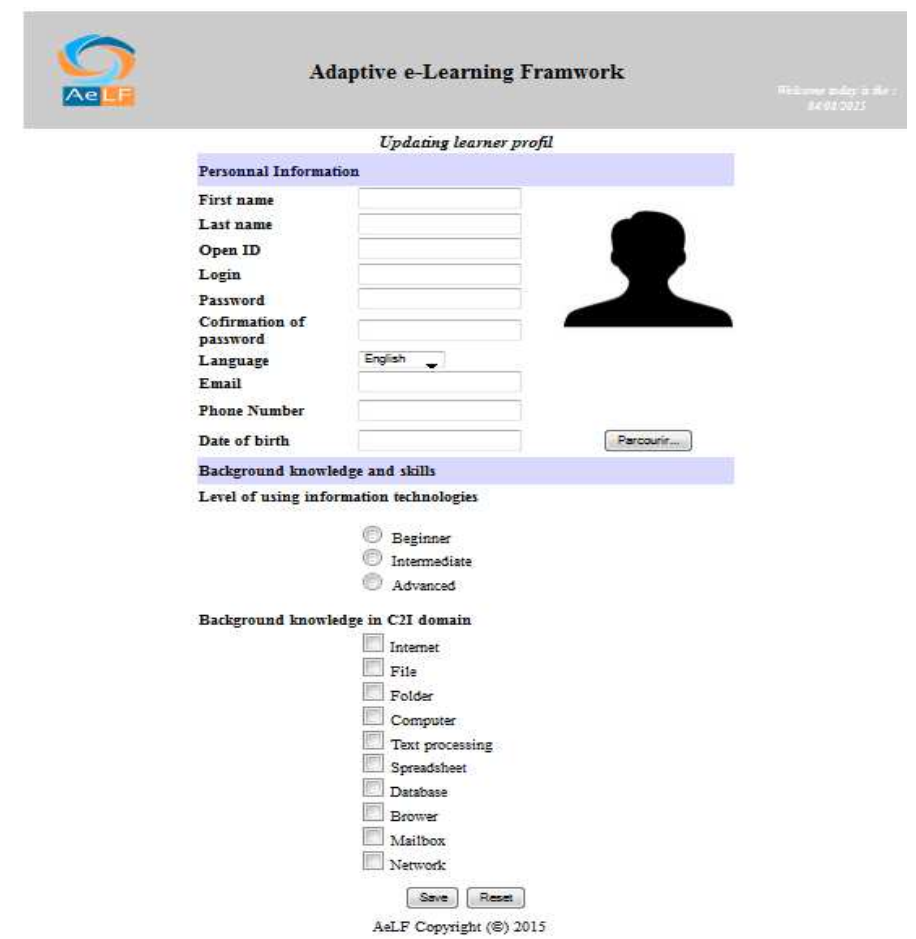

Fig. 3. The AUM updating learner profil.

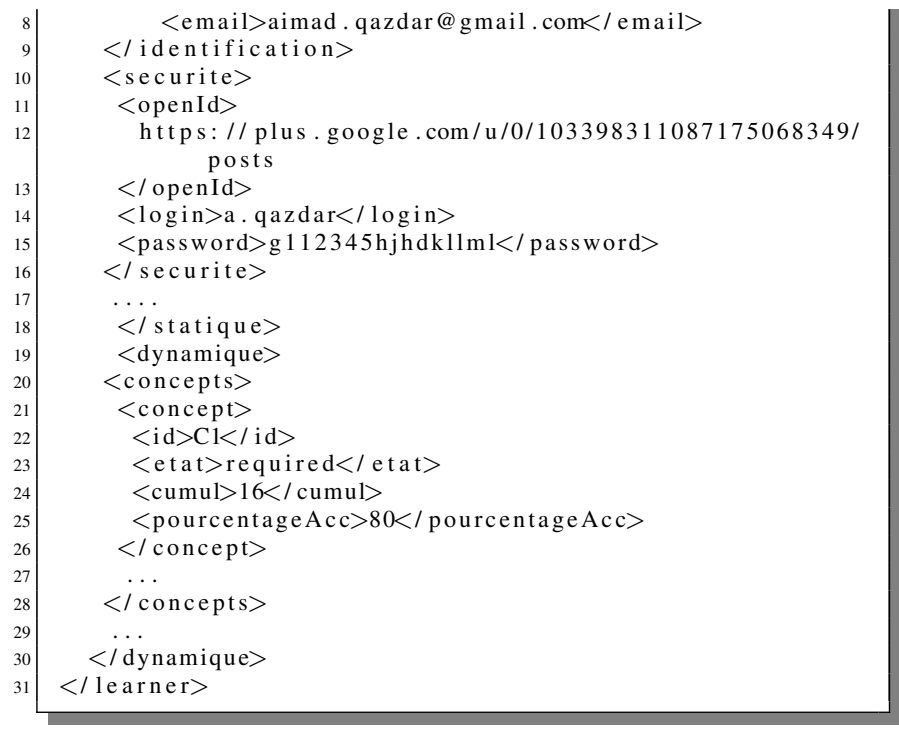

\subsection{The AeLF Domain Model}

The AeLF Domain Model (ADM) is described by its faculty in the representation of concepts to learn, the resources available to learners and the structuring of several elements of the domain. The $\mathrm{ADM}$ has been separated into three parts:

- Content Repository: it contains resources that deal with domain concepts. These resources can be presented as an introduction, definition, example, exercise, paragraph, comment, etc. Each of these resources is described by a single multimedia brick 


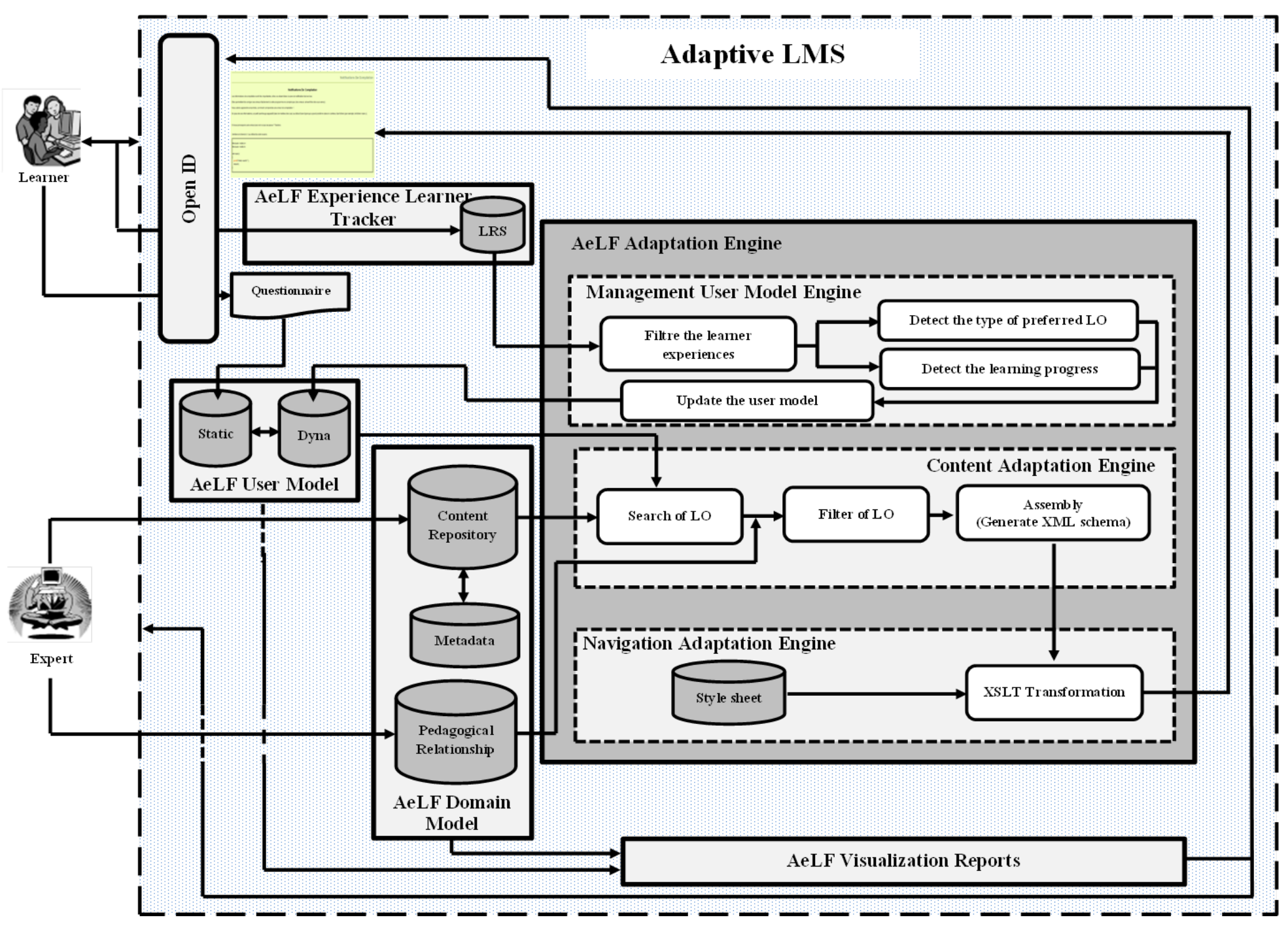

Fig. 2. The overall AeLF infrastructure.

and can be presented in different formats: text, image, sound, video, simulation, and animation.

-Meta-Data : it stores various metadata to describe the different fragments. We used some elements of the LOM standard. Among these metadata we quote: id, title, language, format description, keyword, type of interactivity (active, transmissive, collaborative, communicative, not defined), the level of difficulty (easy, medAUM, hard), semantic density (very low, low, average, high, very high ) and learning time etc.

-Pedagogical Relationship : it stores all relationship between concepts. We chose to use two most important relationships :

-IS-PREREQUISITES-OF: the prerequisites indicate relationship such as the acquisition of a concept $\mathrm{B}$ is subjugated to the mastery of the concept A that precedes it.

-IS-COMPOSED-OF : the composition relationship means that the description of the parent node is performed with the aid of the description of its components. Example a concept A is composed of the following concepts B, C and D.
As output, of the form (Figure4, an XML file is generated. We present follow a part of the metadata XML file.

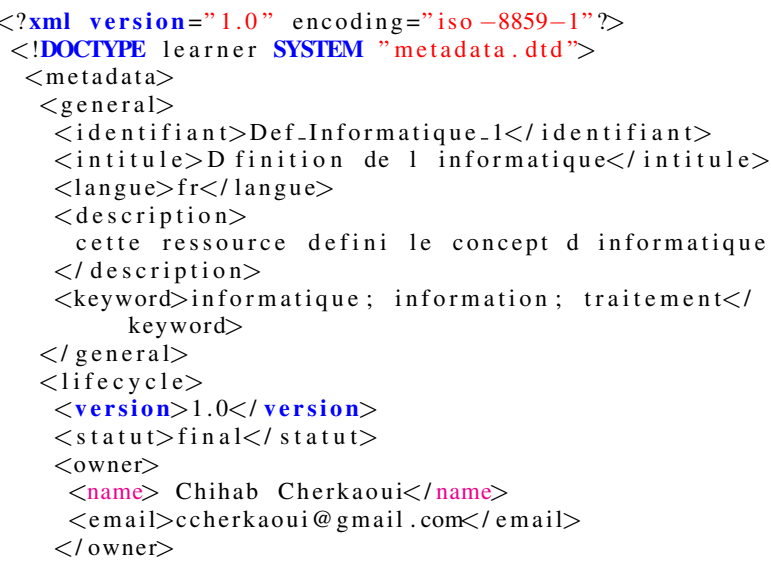



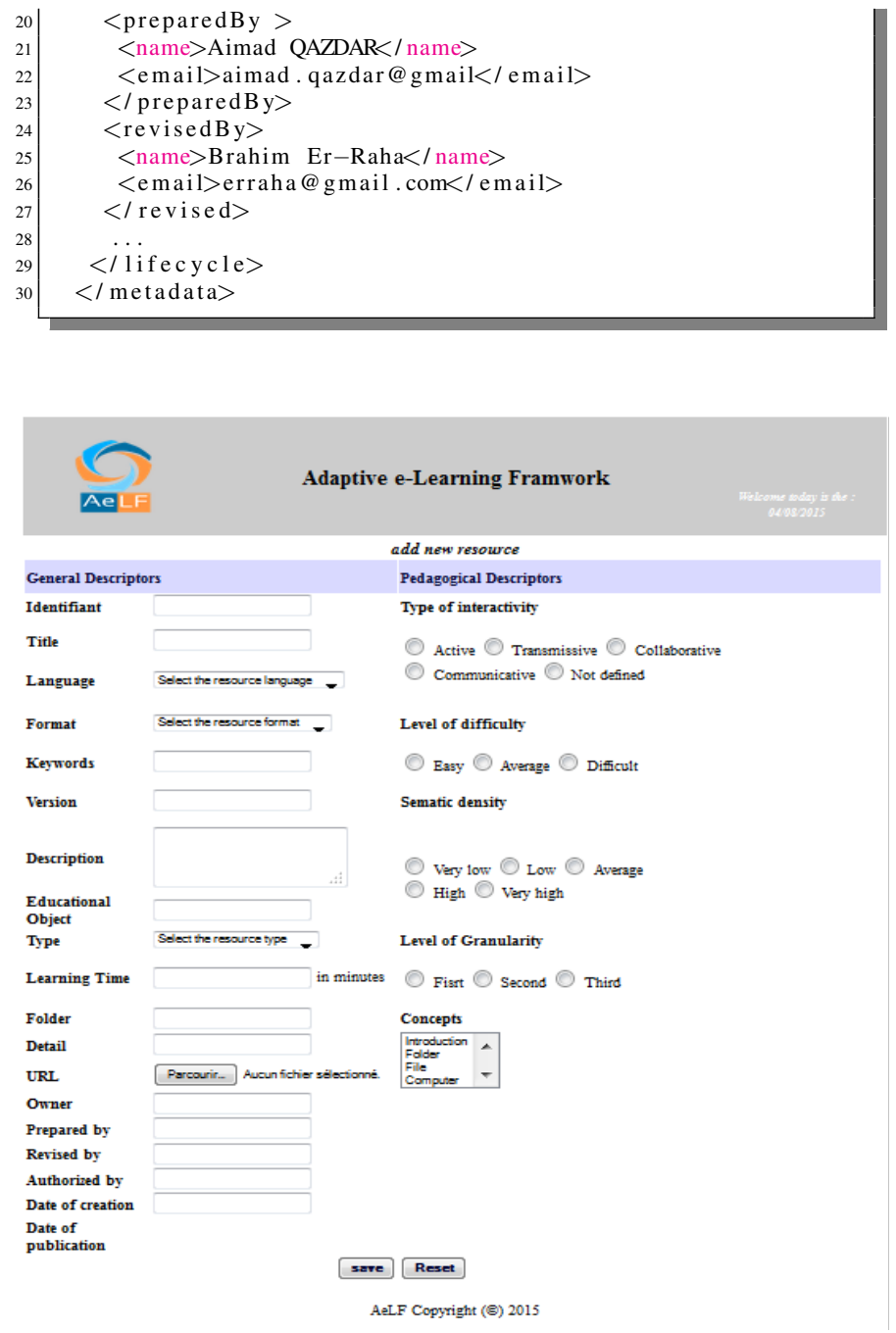

Fig. 4. The ADM editing learning ressource metadata.

\subsection{The AeLF Experience Learner Tracker}

The main role of the AeLF Experience Learner Tracker (AELT) is to catch all the interaction between the learner and the LMS. The AELT is based on the standards XAPI. The xAPI describes the interaction as statements. Every single is comprised of an actor, an action, and an object. I (actor) did (action) this (object). All these statements will be stored in LRS (Learning Recording system) [32]. All information recorded in the LRS will be operated by the Adaptive Engine to update automatically the dynamic part of the AUM.

\subsection{The AeLF Adaptation Engine}

The AeLF Adaption Engine (AAE) is the core of the AeLF. It deals with the automatic production of adaptive content that will be presented later to the learner through the LMS interface. This component has three sub-components: (1) the Management User Model Engine (MUME), (2) the Content Adaptation Engine (CAE) and (3) the Navigation Adaptation Engine (NAE). Every single contains a set of rules to enable the adaptation:
- The Management User Model Engine (MUME): the role of this component is to filter the experiences of the learner, to detect the types of favorite OP and the learning progress. Finally it permits to update the dynamic part of the AUM.

- The Content Adaptation Engine (CAE): this component is used to produce individualized content based on the AUM of each learner. It allows providing a similar content, additional content, and alternating or hiding the contents. First of all it allows searching the LO based on their metadata and the AUM. Then it filters the LO based on predefined pedagogical relationships. The filter process is explained in Figure 5

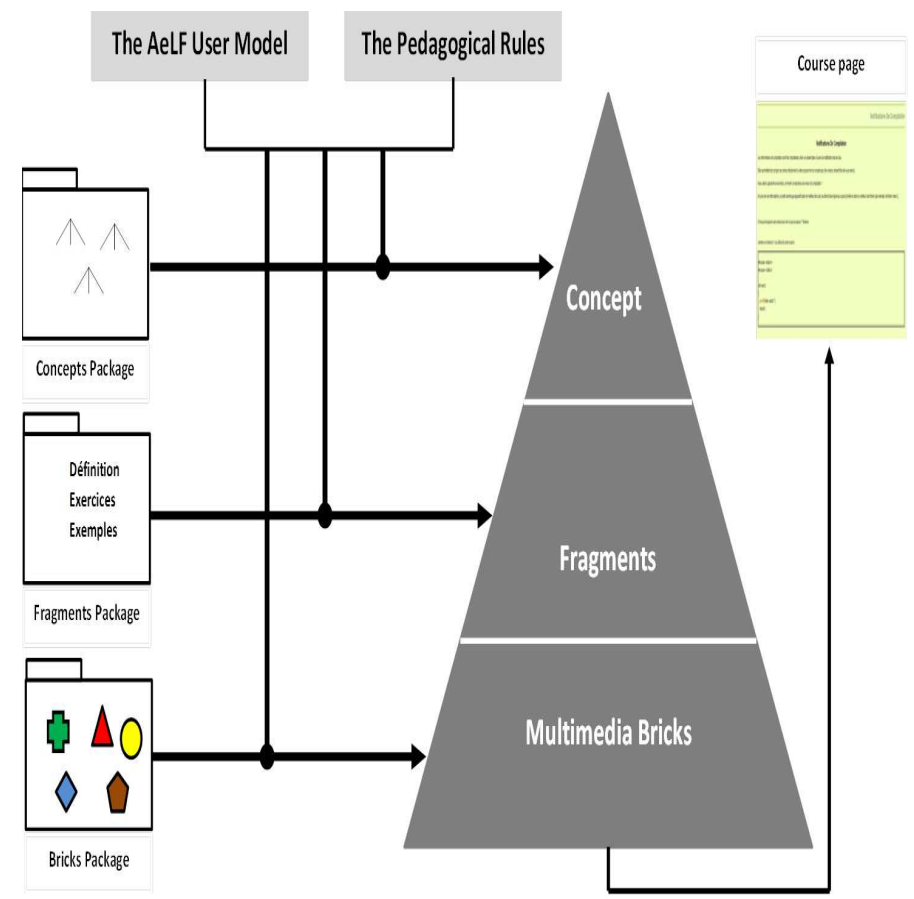

Fig. 5. The assembling courses process.

Finally the CAE organizes and brings these LOs in an XML file that will be transferred later to the Navigation Adaptative Engine.

- The Navigation Adaptation Engine (NAE): by combining the XML file with style sheets the presentation of the link anchors can be adapted. This realizes the direct guidance, the link annotation, and it can also realize the link hiding. Although NAE can use any number of arbitrary colors we typically use the color red to indicate non-recommended link, green for the recommended unvisited link, gray for the recommended visited and yellow for the current link.

\subsection{The AeLF Visualization Report}

The interaction of users with AeLF through the LMS interface results in large amounts of data that can be visualized for both authors and learners. The AeLF Visualization Report (AVR) is a part of the AeLF which is used by teachers and learners to view progress reports in order to observe learners progress and understand the learning processes. The AVR is not described in this paper. 
As AeLF aims at supporting life-long learning it provides an architecture through which different LMSs can interact with a distributed user model (AUM. AUM is used by AAE as an extra source of user information on which adaptation is based. The top left of Figure 2 shows the Open ID identity provider ${ }^{12}$ which is not developed within the AeLF project. In order to have life-long learning support the learner should have an identity that can be used everywhere: on different LMSs and on AeLF. Moving to a different university, an institute or a company can be done without losing the user model stored in the shared AUM service.

When a learner interacts for the first time with the system, the list of concepts learned in the AUM is empty. The concepts stored in the IDM without preconditions and have not been acquired by the learner will initialize the list of active concepts. This allows deciding on the objective of the session. Some elements of the AUM can impact this decision. This impact comes from the background knowledge and skills of the learner represented in the AUM like use of information technology level (beginner, intermediate, Expert), or the background knowledge built from a set of concepts about the domain in study, such as the C2I domain (file, folder, computer, text processing , spreadsheet, database, navigator, and mailbox, etc..). Each decision is controlled by some pedagogical rules.

The selection of one or more concept(s) related with other information depends in particular on the representations of the learner, especially the sequence that will be derived after in fragments. If, for example, the AUM indicates that the learner prefers to study by examples, the sequence will contain more examples. For exercises, the difficulty level will rely on information extracted from the AUM corresponding to her/his level (Beginner, Intermediate, and Advanced). This sequence corresponds to a prototypical sequence of fragments to complete the selected learning concept. Depending on the AUM, the system associates a multimedia brick with each fragment of this sequence. If the AUM indicates for example that the learner prefers pictures and videos, the system will promote anything that is multimedia. If she/he prefers reading on the screen, the text associated with fragments will be used to create a course page.

\section{CONCLUSION AND FUTURE WORK}

As the e-learning platforms (LMS and MOOC) have become used more and more as learning environments in several educational institutes, the lack of adaptation in these systems has become an issue.

In this paper, we showed the structure of AeLF as a new framework of adaptivity in LMSs. We also described all its components and features like Single Sign-On. Finally we explained the process used in AeLF to turn a static course in LMS to an adaptive course.

It is clear that to arrive at the expected system several issues remain to be addressed. Our proposed solution continues along these lines to try to finish a first functional prototype which will be tested and validated.

\footnotetext{
${ }^{12} \mathrm{http}: / /$ openid.net/
}

\section{References}

[1] Battou Amal, El Mezouary Ali, Cherkaoui Chihab, and Mammass Driss. Towards an adaptive learning system based on a new learning object granularity approach. International Journal of Advanced Computer Science and Applications(IJACSA), 2, 2011.

[2] Scherl Andre. Hypermediale Wissensvernetzung in Physiologie und Physik, Entwicklung einer webbasierten Lernumgebung und Untersuchung ihres Einfusses auf interdisziplinres Lernen. PhD thesis, dem Physiologischen Institut der Ludwig Maximilians Universitt Mnchen, 2013.

[3] Amal Battou. Approche granulaire des objets pdagogiques en vue de ladaptabilit dans le cadre des Environnements Informatiques pour l'Apprentissage Humain. $\mathrm{PhD}$ thesis, Facult des Sciences Agadir, Universit Ibn zohr, 2012.

[4] Paul De Bra, Ad Aerts, Bart Berden, Barend de Lange, Brendan Rousseau, Tomi Santic, David Smits, and Natalia Stash. Aha! the adaptive hypermedia architecture. In Proc. of the fourteenth ACM conference on Hypertext and Hypermedia, pages 81-84. ACM Press, 2003.

[5] Peter Brusilovsky. Methods and techniques of adaptive hypermedia. User Modeling and User-Adapted Interaction, 6(23):87-129, 1996.

[6] Peter Brusilovsky. Adaptive hypermedia. User Modeling and User-Adapted Interaction, 11(1-2):87-110, 2001.

[7] Peter Brusilovsky, John Eklund, and Elmar Schwarz. Webbased education for all: A tool for development adaptive courseware. In Computer Networks and ISDN Systems, pages 1-7, 1998.

[8] Owen Conlan and VincentP. Wade. Evaluation of apels an adaptive elearning service based on the multi-model, metadata-driven approach. In PaulM.E. De Bra and Wolfgang Nejdl, editors, Adaptive Hypermedia and Adaptive WebBased Systems, volume 3137 of Lecture Notes in Computer Science, pages 291-295. Springer Berlin Heidelberg, 2004.

[9] Paul De Bra and David Smits. A fully generic approach for realizing the adaptive web. In Mria Bielikov, Gerhard Friedrich, Georg Gottlob, Stefan Katzenbeisser, and Gyrgy Turn, editors, SOFSEM 2012: Theory and Practice of Computer Science, volume 7147 of Lecture Notes in Computer Science, pages 64-76. Springer Berlin Heidelberg, 2012.

[10] Paul De Bra, David Smits, and Natalia Stash. The design of aha! In Proceedings of the Seventeenth Conference on Hypertext and Hypermedia, HYPERTEXT '06, pages 133-134, New York, NY, USA, 2006. ACM.

[11] Paul De Bra, David Smits, Kees van der Sluijs, AlexandraI. Cristea, Jonathan Foss, Christian Glahn, and ChristinaM. Steiner. Grapple: Learning management systems meet adaptive learning environments. In Alejandro Pea-Ayala, editor, Intelligent and Adaptive Educational-Learning Systems, volume 17 of Smart Innovation, Systems and Technologies, pages 133-160. Springer Berlin Heidelberg, 2013.

[12] Marijana Despotovic-Zrakic, Aleksandar Markovic, Zorica Bogdanovic, Dusan Barac, and Srdjan Krco. Providing adaptivity in moodle lms courses. Educational Technology \& Society, 15(1):326-338, 2012.

[13] Sidney D'Mello, Scotty Craig, Karl Fike, and Arthur Graesser. Responding to learners cognitive-affective states with supportive and shakeup dialogues. In JulieA. Jacko, editor, Human Computer Interaction. Ambient, Ubiquitous and 
Intelligent Interaction, volume 5612 of Lecture Notes in Computer Science, pages 595-604. Springer Berlin Heidelberg, 2009.

[14] Moushir M. El-Bishouty, Hiroaki Ogata, and Yoneo Yano. Personalized knowledge awareness map in computer supported ubiquitous learning. In Proceedings of the Sixth IEEE International Conference on Advanced Learning Technologies, ICALT 06, pages 817-821, Washington, DC, USA, 2006. IEEE Computer Society.

[15] S. Graf and Kinshuk. Adaptive technologies. In In Spector, J.M. Merrill, M.D. Elen, J. Bishop, M.J. (Eds.), Handbook of Research on Educational Communications and Technology, pages 771-779, 2014.

[16] S. Graf and B. List. An evaluation of open source e-learning platforms stressing adaptation issues. pages 163-165, July 2005.

[17] Sabine Graf and Cindy Ives. A flexible mechanism for providing adaptivity based on learning styles in learning management systems. In in Proceedings of the IEEE International Conference on Advanced Learning Technologies (ICALT 2010), IEEE Computer Society, pages 30-34, 2010.

[18] Sergio Gutierrez-Santos, Abelardo Pardo, and Carlos Delgado Kloos. Authoring courses with rich adaptive sequencing for ims learning design. j-jucs, 14(17):2819-2839, sep 2008.

[19] Surjono Herman Dwi. The design of adaptive e-learning system based on students learning styles. International Journal of Computer Science and Information Technologies (IJCSIT), 2011.

[20] Geert-Jan Houben, Keesvan der Sluijs, Peter Barna, Jeen Broekstra, Sven Casteleyn, Zoltn Fiala, and Flavius Frasincar. Hera. In Gustavo Rossi, Oscar Pastor, Daniel Schwabe, and Luis Olsina, editors, Web Engineering: Modelling and Implementing Web Applications, Human-Computer Interaction Series, pages 263-301. Springer London, 2008.

[21] Gwo-Jen Hwang, Tzu-Chi Yang, Chin-Chung Tsai, and Stephen J.H. Yang. A context-aware ubiquitous learning environment for conducting complex science experiments. Computers and Education, 53(2):402 - 413, 2009.

[22] Jantke K P. and Schulz A. Adaptivity in moodle beyond the limits of adaptivity in moodle., 2011.

[23] Pythagoras Karampiperis and Demetrios Sampson. Adaptive learning resources sequencing in educational hypermedia systems. Educational Technology and Society, 8:128-147, 2005.

[24] Evgeny Knutov, Paul De, and Bra Mykola Pechenizkiy. Ah 12 years later: a comprehensive survey of adaptive hypermedia methods and techniques. New Rev. Hypermedia Multimedia, 2009.

[25] S. Martn, E. Sancristobal, R. Gil, M. Castro, and J. Peire. Mobility through location-based services at university. International Journal of Interactive Mobile Technologies (iJIM), 2(3), 2008.

[26] Violeta Moisa. Adaptive learning management system. Journal of Mobile, Embedded and Distributed Systems, 5(2), 2013.

[27] Fabio Paterno. Designing web user interfaces adaptable to different types of use. museums and the web, 1999.

[28] E. Popescu. Adaptation provisioning with respect to learning styles in a web-based educational system: an experimental study. Journal of Computer Assisted Learning, 26(4):243257, 2010.
[29] Graf Sabine. Adaptivity In Learning Management Systems Focussing On Learning Styles. $\mathrm{PhD}$ thesis, Vienna University Of Technology ,Germany, 2007.

[30] OlgaC. Santos, Carmen Barrera, and JessG. Boticario. An overview of alfanet: An adaptive ilms based on standards. In PaulM.E. De Bra and Wolfgang Nejdl, editors, Adaptive Hypermedia and Adaptive Web-Based Systems, volume 3137 of Lecture Notes in Computer Science, pages 429-432. Springer Berlin Heidelberg, 2004.

[31] Gert Sauerstein. KI-Anstze zur Lerner-Adaption in LernManagement-Systemen. $\mathrm{PhD}$ thesis, Technische Universitt Ilmenau,Germany, 2007.

[32] Foreman Steve. The xapi and the lms: What does the future hold?, 102013. http://www.learningsolutionsmag.com/articles/1271/thexapi-and-the-lms-what-does-the-future-hold.

[33] Judy C.R. Tseng, Hui-Chun Chu, Gwo-Jen Hwang, and ChinChung Tsai. Development of an adaptive learning system with two sources of personalization information. Computers and Education, 51(2): 776 - 786, 2008.

[34] Declan Dagger Vincent Wade. Personalisation for all: Making adaptive course composition easy. In In Special Edition of the Educational Technology and Society Journal, IEEE IFETS, pages 9-25, 2005.

[35] Beverly Woolf, Winslow Burleson, Ivon Arroyo, Toby Dragon, David Cooper, and Rosalind Picard. Affect aware tutors recognising and responding to student affect. Int. J. Learn. Technol., 4(3/4):129-164, October 2009.

[36] Yao Jung Yang and Chuni Wu. An attribute-based ant colony system for adaptive learning object recommendation. Expert Systems with Applications, 36(2, Part 2):3034 - 3047, 2009. 